\title{
Evaluation of Template Releasing Energy in Nanoimprint Lithography
}

\author{
Tomoki Nishino $^{1,2}$, Fujita Honoka ${ }^{1}$, Hiroaki Kawata ${ }^{1,2}$ and Yoshihiko Hirai ${ }^{1,2}$ \\ ${ }^{1}$ Osaka Prefecture University \\ Department of Physics and Electronics Engineering, Graduate School of Engineering \\ 1-1 Gakuen-cho, Nakaku, Sakai, Osaka, 599-8531 Japan \\ ${ }^{2}$ Japan Science and Technology Agency \\ CREST(Core Research of Evolutional Science \& Technology) \\ 4-1-8, Honcho, Kawaguchi-shi, Saitama 332-0012 Japan
}

\begin{abstract}
To investigate template releasing process in nanoimprint lithography, template releasing energy from resist polymer is evaluated under various conditions using test rig. The template releasing energy is proportional to the surface energy of the template, however it does not depend on rigidness of the system and template lifting speed.
\end{abstract}

Keywords: nanoimprint, adhesion, releasing energy, surface energy, template

\section{Introduction}

In nanoimprint lithography[1,2], adhesion and/or friction between template and resist is important issues in template releasing process, because the resist to might be peeled off from the substrate or fractured, which causes fetal defects. Those defects in the template releasing process directory impact on process yield and crucial importance in industrial applications.

There have been several reports on eliminating defects by applying an anti-sticking coating on the template surface[3] or resist material. There have been several reports on evaluation of anti-sticking agents or resist materials by measuring the releasing forces when a template is detached (removed) from a resist polymer. In these studies, the releasing forces are mostly evaluated by the template releasing forces.[4,5] However, quantitative measurement of the template releasing forces is very difficult because the absolute values strongly depend on rigidly of each equipment and/or insufficient holding of the template and substrate, etc.

On the other hand, evaluation of anti-sticking layer or resist materials has largely been confined to reports on the contact angle for water droplets on the surface of these materials, and only a few reports have attempted template releasing materials or method based on mechanical point of view. Based on fracture mechanics[6] which is also used for such purposes as evaluating the adhesion of silicon on insulator (SOI) wafers, Taniguchi[7] et al. observed the cracks between resist and template to extract releasing energy to be required to cleave them apart. Using a similar method, Houle[8] and S. Garidel et al.[9] reported on the quantitative evaluation of surface energy for releasing agents and UV resists, and discussed the differences in releasing energy arising from the resist composition and template releasing direction. Also, Landis et al.[10] measured the fracture stress in various crack directions against patterned template. However, the previous works did not take care of process conditions such as relations between surface free energy and releasing energy.

In this study, we developed the template release equipment which evaluated displacement and load for releasing and in-site observe the releasing process. Using the system, we evaluated the releasing energy for template releasing process.

\section{Experiment Procedure}

Figure 1 shows a schematic diagram of the test rig for template releasing energy. A resist is coated 
on the template and a quartz plate is put on the resist. By UV exposure, the resist is glued on the quartz plate. Then the quartz plate is lifted up by rod, which are placed $60 \mathrm{~mm}$ from the center of the template in an axial symmetry.

The displacement of the quartz plate is measured by optical sensors (Keyence Si-F01), which are placed $30 \mathrm{~mm}$ from the center of the template. The nominal resolution is $1 \mathrm{~nm}$.

The loads on the lifting rods are measured using conventional load cells (Minebea CB17-3K-11). To eliminate the energy loss at the side wall of the template, the edge of the template is tapered by 45 degree chamfering.

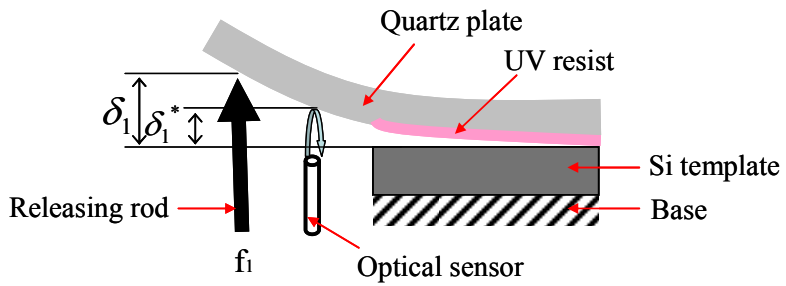

Figure 1. Schematic diagram of the test rig

\section{Evaluation of template releasing energy}

In this report, the template releasing energy $G_{\text {de-molding }}$ is defined as the required energy per unit area to release a resist from a template. On the basis of a classical fracture theory ${ }^{8)}$, the surface energy $\gamma$ is expressed as being one-self half of the fracture energy $G_{c}$ per unit area to create a new surface owing to crack propagation. We extract the fracture energy $G_{c}$ as the template releasing energy $G_{d e-\text { molding }}$ to separate the resist from the template, as defined in the following equation :

$$
G_{\text {de-molding }} \equiv G_{c}=2 \gamma
$$

Figure 3 illustrates a diagram of crack propagation between the template and the resist. The quartz plate is stable position at displacement $\delta_{\text {start }}$ and load $f_{\text {start }}$, as shown in Fig. 2 (a). When additional load is applied, the crack grows to a new stable length, as illustrated in Fig. 2 (b), where the displacement is $\delta_{\text {end }}$ and the load is $f_{\text {end }}$. The amount of strain energy in the quartz plate $U_{\text {elastic,start }}$ before crack propagation and the externally applied energy $U_{\text {external }}$ balances the amount of strain energy in the quartz plate

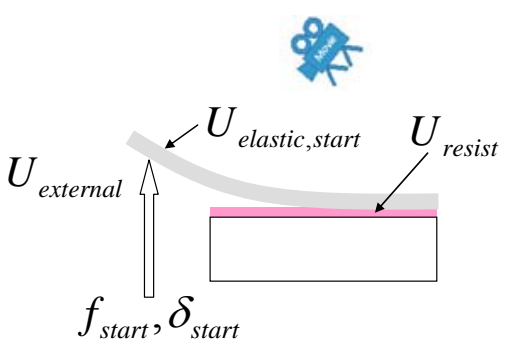

a) The state that is in the middle of releasing.

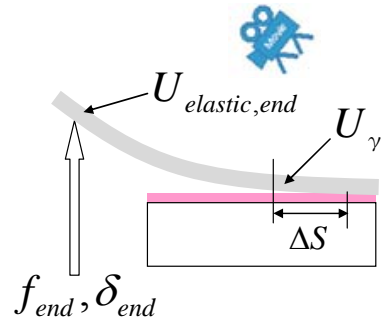

b) The state that proceeding of releasing.

Figure 2. Schematic of crack propagation in releasing process

$U_{\text {elastic,end }}$ after crack propagation, the elastic energy consumption in the resist $\Delta U_{\text {resit }}$ and the amount of energy consumption for template releasing $U_{d e-\text { molding }}$ as expressed by

$U_{\text {elastic,start }}+U_{\text {external }}$

$=U_{\text {elastic,end }}+U_{\text {de-molding }}+\Delta U_{\text {resist }}$

The strain emerging at $U_{\text {elastic,start }}$ and $U_{\text {elastic,end }}$ are expressed as follows:

$U_{\text {elastic,start }}=\frac{1}{2} f_{\text {start }} \delta_{\text {start }}$

$U_{\text {elastic,end }}=\frac{1}{2} f_{\text {end }} \delta_{\text {end }}$

The energy $U_{\text {external }}$ is given by sum of integration of the applied load $f_{n}$ and displacement $\delta_{n}$ of each rod:

$U_{\text {external }}=\int_{\delta_{\text {start }}}^{\delta_{\text {end }}} f d \delta$

$\Delta U_{\text {resist }}$ is derived under assumption that the resist is stretched in nominal direction as follows:

$\Delta U_{\text {resist }} \approx \frac{1}{2} \cdot\left(\frac{f_{e}{ }^{2}}{S_{e} E}-\frac{f_{s}{ }^{2}}{S_{s} E}\right) \cdot d$

where $E$ and $d$ are the elastic modulus and initial thickness of the resist, respectively. $S_{e}$ and $S_{s}$ are the adhesion areas between the resist and 
plate before and after crack propagation.

To qualify the external energy $U_{\text {external }}$, we need the displacement $\delta$ at the lifting rod. However, the displacement of the quartz plate $\delta^{*}$ is measured by the optical sensor, as shown in Fig. 1. We estimate the displacement $\delta$ at the lifting rod by numerical calculation using the finite element method (FEM) based on the displacement $\delta^{*}$ at the optical sensor position, as demonstrated in Fig. 3 . The correction factor in the peeling mode is 0.32 . As a result, the template releasing energy per unit area $G_{d e-\text { molding }}$ is derived as follows:

$$
\begin{aligned}
& G_{\text {de-molding }}=U_{\text {demolding }} / \Delta S \\
& =\left(U_{e s}+U_{\text {external }}-U_{e e}-\Delta U_{\text {resist }}\right) / \Delta S
\end{aligned}
$$

where $\Delta S$ is the newly revealed fresh surface area according to the releasing process.

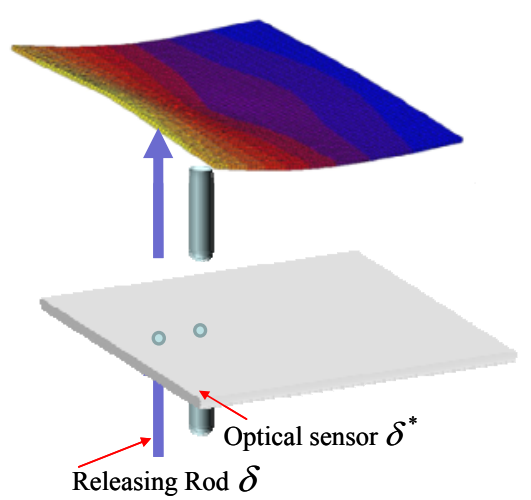

Figure 3. Estimation of the displacements of quartz plate.

\section{Experimental process and conditions}

A template is bonded on to the base unit, and a UV-curable resist polymer is coated on it. To promote successful releasing without sticking defects, we use OPTOOL-DSX(Daikin Industries) as an anti-sticking layer on the template surface. The template size is $10 \mathrm{~mm}^{2}$, and the quartz plate is $150 \mathrm{~mm}^{2}$ and $2 \mathrm{~mm}$ in thickness.

The resist is PAK-01(TOYOGOSEI), which is a solvent-soluble polymer for UV nanoimprinting. The initial thickness of the resist is typically $10.0 \mu \mathrm{m}$. A quartz plate as a substrate is put on the resist and cured by UV exposure through the quartz plate. The surrounding atmosphere was purged with nitrogen to eliminate oxygen inhabitance for UV curing. After the resist is cured, the resist is released from the Si template by lifting the quartz plate up with rods.

Cracks between the template and the resist are observed by using a video camera to investigate crack propagation in the releasing process, as shown in Fig. 4.

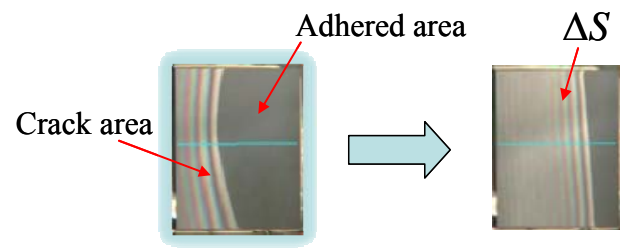

Figure 4. Observation of the releasing

\section{Experimental result}

Template releasing energy between resist and template are investigated in variation with process conditions such as system rigidly, template surface energy, template releasing speed under the peeling mode.

\subsection{Dependence on system rigidness}

To verify the test rig and extraction procedure of template releasing energy, we examined the dependence on the system rigidness by alternating the thickness of the quartz plate as one of the components of the system. The thickness of the quartz plates are varied from 2 to $6 \mathrm{~mm}$.

Figure 5(a) shows the relation between the rod displacement and load for various thicknesses of the quartz plate to examine the stability of the rigidness of the system. As the thickness of the quartz plate increases, which means the rigidly increases, the releasing force increases and the displacement decreases. Figure 5(b) shows the extracted surface energy between the resist and template. The lifting speed of the rod was 1 $\mu \mathrm{m} / \mathrm{sec}$. The template releasing energy between the resist and template does not depend on the thickness of the quartz plate, which means the releasing energy is independently evaluated on rigidness of the system.

\subsection{Dependence on surface energy of template}

To assist successful releasing, we usually use OPTOOL-DSX as an anti-sticking layer to decrease the surface energy of the template To verify the dependence of the template releasing energy on the template surface energy, the template surface energy is modified by UV Ozone exposure to be 804,645 and $302 \mathrm{~mJ} / \mathrm{cm}^{2}$, which are equivalent to contact angles for water droplet of $20^{\circ}, 60^{\circ}$ and $100^{\circ}$, respectively. We use virgin templates under nitrogen ambient to 
eliminate Ozone generation and oxygen inhabitation for UV resist. The lifting speed of the rods was $1.0 \mu \mathrm{m} / \mathrm{sec}$.

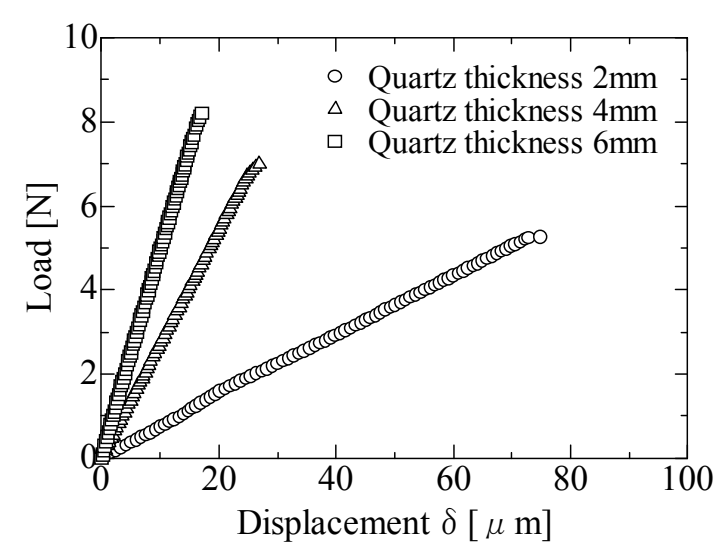

a) Displacement-force curve.

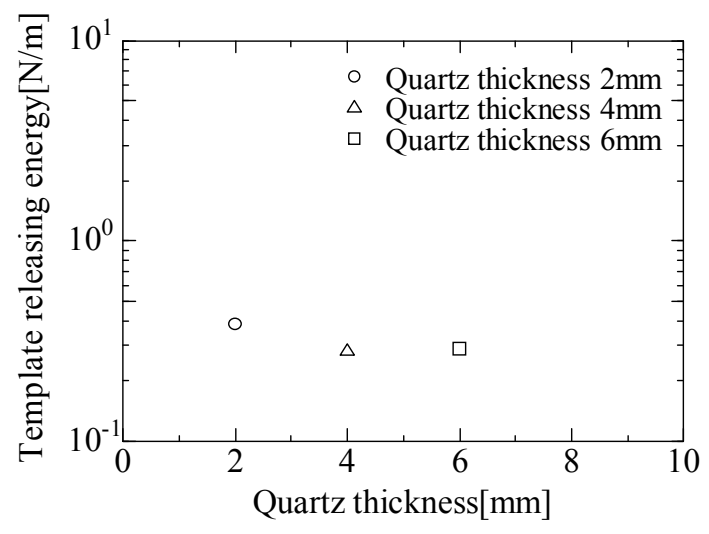

b) Template releasing energy vs. thickness of the quartz plate

Figure 5. Dependence of template releasing energy on the quartz thickness. (Rod lifting speed: $1 \mu \mathrm{m} / \mathrm{sec}$, Contact angle for water droplet on the template: $88^{\circ}$ )

Figure 6(a) shows the relation between the rod 1 displacement and load in variation with the contact angle $\theta$ for water droplet on the template. As the contact angle decreases (increment of the surface energy of template), the displacement and releasing force increase.

Figure 6(b) shows the relation between the surface energy of the template and the template releasing energy. The template releasing energy is proportional to the surface energy of the template, which is reasonable result.

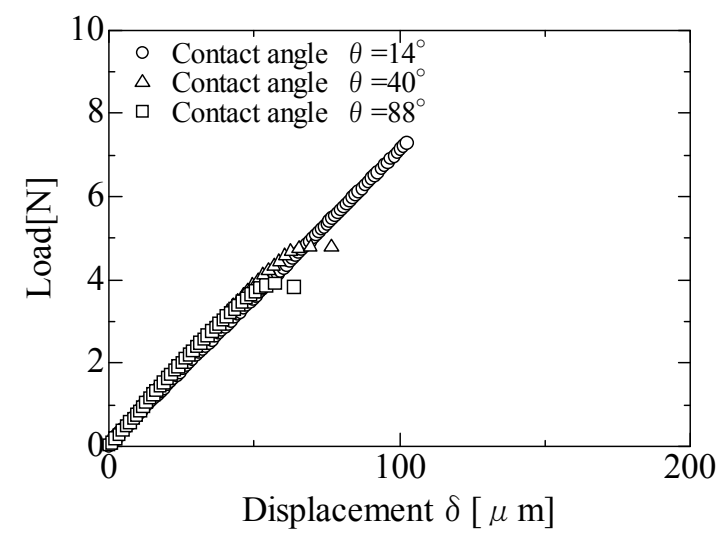

a) Displacement-force curve

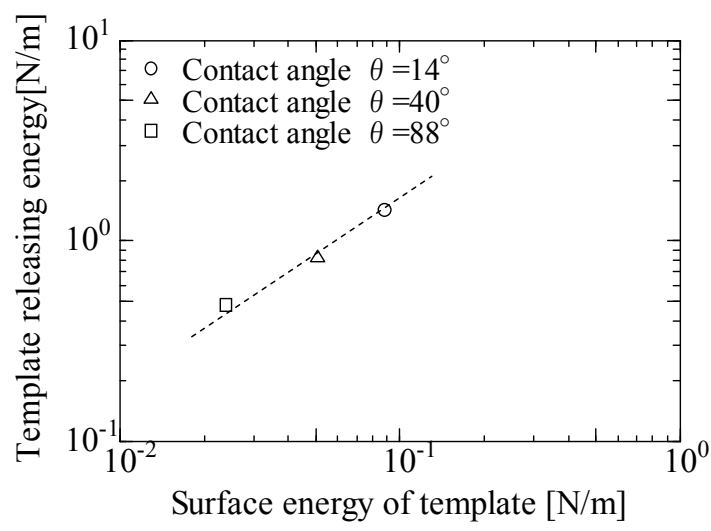

b) Template releasing energies in variation with surface energy of the template. Rod lifting speed: $1 \mu \mathrm{m} / \mathrm{sec}$, Quartz thickness: 2mm)

Figure 6. Dependence of template releasing energy on surface energy of template

\subsection{Dependence on lifting speed}

One of the interesting issues is the lifting speed whether slow lifting speed is better or not. The lifting speed of the rods is varied from $500 \mathrm{~nm} / \mathrm{sec}$, to $10 \mu \mathrm{m} / \mathrm{sec}$ to investigate dependency on the lifting speed.

Figure 7(a) shows displacement and load of the lifting rod in various lifting speeds. As the rod lifting speed increases, the template releasing load increases. However, the template releasing energy does not depend on the lifting speed of rod as shown in Fig. 7(b). In other word, the template releasing energy does not depend on the lifting speed, which makes sense from fracture dynamics viewpoint that the template releasing events are 


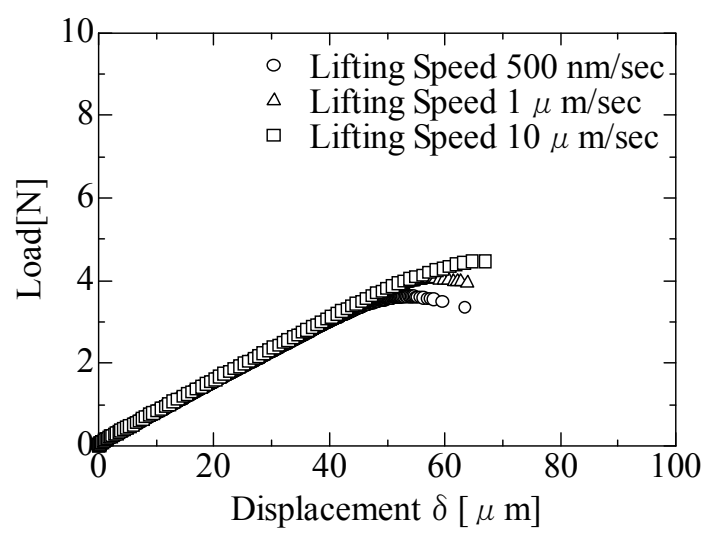

a) Displacement-force curve

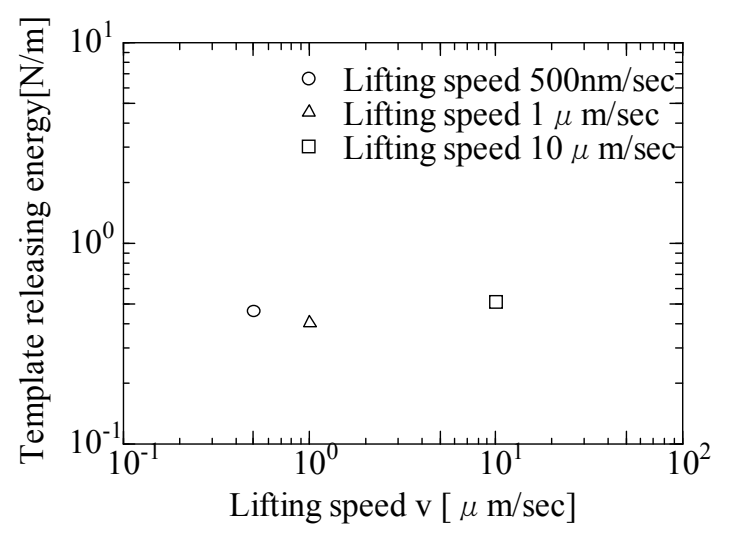

b) Template releasing energy

Figure 7. Dependence of surface energy on lifting speed of the rod. (Quartz thickness:2.0 $\mu \mathrm{m}$, Contact angle for water droplet on the template: $88^{\circ}$ )

related to the template releasing energy. However, the velocity is so slow in our experiment and further investigations should be done in high speed or impact releasing.

\section{Conclusion}

In nanoimprint lithography, template releasing energy between the resist and template is verified in template releasing process. The system evaluates the template releasing energy regardless of the rigidness of a system. The template releasing energy is proportional to the surface energy of the template, however it does not depend on the releasing speed. These results helps understanding template releasing phenomena in quantitatively to evaluate the template releasing energy required for template releasing rather than the template release force.

\section{References}

1. S. Y. Chou, P. Krauss, and P. Renstrom, J. Vac. Sci. Technol., B14 (1996), 4129.

2. M. Colburn, S. Johnson, M. Stewart, S. Damle, T. Bailey, B. Choi, M. Wedlake, T. Michaelson, S. Sreenivasan, J. Ekerdt, and C. Willson, Proc. SPIE 3676 (1999), 378.

3. Y. Hirai, S. Yoshida, A. Okamoto, Y. Tanaka, M. Endo, S. Lrie, H. Nakagawa and M. Sasago, J. Photopolymer Sci. Technol., 14 (2001), 457.

4. V. Trabadelo, H. Schift, S. Merino, S. Bellini and J. Gobrecht, Microelectron. Eng., 85 (2008), 907.

5. Y. Guo, G. Liu, X. Zhu and Y. Tian, Microsyst. Technol. 13 (2007), 411.

6. A. Griffith, Philosophical Transactions of the Royal Society of London. A221 (1921), 163.

7. J. Taniguchi, K. Takeshi, T. Yuji, K. Yasuo, M. Iwao, K. Masanori, H. Hiroshi, S. Nobuji and T. Kentaro, Jpn. J. Appl. Phys. Part 141 (2002), 4194.

8. F. A. Houle, E. Guyer, D. C. Miller and R. Dauskardt, J. Vac. Sci. Technol., B 25 (2007), 1179.

9. S. Garidel, M. Zelsmann, N. Chaix, P. Voisin, J. Boussey, A. Beaurain and B. Pelissier, J. Vac Sci. Technol., B 25 (2007), 2434.

10. S. Landis, N.Chaix, C. Gourgon and T. Leveder, NanoTechnology 19 (2008), 125305 . 